\title{
Multiple resonance capture of a compound system under harmonic excitation
}

\author{
Thanh Tung Pham - Claude-Henri Lamarque \\ Alireza Ture Savadkoohi - Stéphane Pernot \\ Université de Lyon \\ École Nationale des Travaux Publics de l'État, DGCB, FRE \\ CNRS 3237, 3 rue Maurice Audin \\ F-69518, Vaulx-en-Velin cedex \\ \{thanh-tung.pham, lamarque, alireza.turesavadkoohi, stephane.pernot\}@entpe.fr
}

\begin{abstract}
Vibration exchange between a master linear oscillator and a slave Nonlinear Energy Sink (NES) under harmonics excitation by means of several resonance capture is investigated and commented upon. The importance of higher frequencies by introducing the concept of the relative mode is pinpointed. A fast and special iterative technique and then the more general form of it is introduced in order to evaluate the system unknowns. Finally, the effect of the correct resonance paring on detecting the actual system behavior is illustrated by an example.

RÉSUMÉ. Nous explorons par des méthodes analytiques et numériques les échanges vibratoires entre un oscillateur maître et un puits d'énergie non linéaire (NES) sous excitations harmoniques, obtenus au moyen de plusieurs captures de résonance. Nous mettons en évidence l'importance des fréquences supérieures via la notion de mode relatif. Pour mener l'étude, deux méthodes itératives l'une simple et rapide, l'autre plus complète sont introduites pour déterminer les solutions des systèmes algébriques obtenus. Enfin, les effets de la résonance correctement évalués sur le comportement du système sont illustrés par un exemple.

KEYWORDS: passive control, resonance capture, NES, relative mode.

MOTS-CLÉS : contrôle passif, résonance capturée, NES, mode relatif.
\end{abstract}

DOI:10.3166/EJCM.20.125-142 (C) 2011 Lavoisier, Paris 


\section{Introduction}

One of the most important aspects in protecting different kinds of structures is to mitigate the seismic induced forces to the system as much as possible; numerous systems have been developed to actively or passively control the structure against dynamic forces during the past decades (Silva, 2005). Most of classical seismic mitigation devices are linear and they require the addition of significant mass to the structure that is in contrary with the novel design criterion which seeks to reduce the total structural mass. Another important issue in the design of such devices is that they should be able to absorb seismic effects for a broad band of frequencies. During the last decade the NES devices or Targeted Energy Transfer (TET) systems have been developed so that the imposed energy to a system is transferred to a strongly nonlinear light attachments in an almost irreversible manner; the mechanism is mainely based on a 1:1 resonance capture. Theoretical background of these systems is quite well developed (Vakakis et al., 2009a), (Vakakis et al., 2009b). Most of the past studies about the NES devices were based on the fact that the main structure remains always on its elastic phase. Schmidt and Lamarque (Schmidt et al., 2010) studied the energy transfer from initial single DoF system including non-smooth term of friction to a NES under free transient or periodic external solicitations. They illustrated that under free excitation, a NES designed for underlying linear elastic oscillator is still efficient for the elasto-plastic case and leads to reduced oscillations in comparison with the behavior without coupled NES. They show that under periodic solicitation and the same design, efficiency of energy pumping can be reduced or destroyed in the whole system, but that it is possible to improve the design by a numerical parametric study to get correct results. The effect of the NES on the steady state dynamics of a coupled system was studied experimentally and theoretically in (Jiang et al., 2003), while more mature studies of these systems under harmonic forcing by considering the bifurcation and stability of fixed points are pointed out by Starosvetsky and Gendelman (Starosvetsky et al., 2008a). The same authors studied quasi-periodic response of this system under external sinusoidal forcing in a vicinity of main (1:1) resonance (Gendelman et al., 2007); they revealed two qualitatively different regimes of the quasi-periodic response: The first appears as a result of linear instability of the steady-state regime of the oscillations. The second one occurs due to interaction of the dynamical flow with invariant manifold of damped-forced nonlinear normal mode of the system, resulting in hysteretic motion of the flow in the vicinity of this mode. Dynamic responses of this compound system under harmonic forcing in the regime of 1:1:1 resonance by detailed investigation of the strongly modulated response which is not related to the fixed points of average modulation equations of the system is investigated in (Starosvetsky et al., 2008b). Recently, Pham et al. (Pham et al., 2011), examined passive control of a single DoF system by a NES under parametric resonance and they compared results of the system with the Tuned Mass Damper (TMD) controller devices. In this study we consider a 2DoF nonlinear system under harmonic excitation and we will explore the system by endowing the idea of relative mode and implementing iterative techniques. Organization of the article is as it follows: 
The mathematical model of the system under consideration is illustrated in Section 2. The global view of the problem and implemented assumptions and techniques for dealing with the system unknowns are defined in Section 3. The 1:1,3 resonance case and the analytical procedure for revealing the behavior of the system are introduced in Section 4; a fast iterative technique is introduced at the same section in order to determine approximations of the absolute values of harmonics. A complete iterative technique for evaluating phases and amplitudes of harmonics is explained in Section 5. Then other types of resonance case by illustrating the importance of correct resonance paring in detecting the system behavior are pinpointed in Section 6 . Conclusion remarks are summarized in Section 7.

\section{Mathematical model of the system under consideration}

Let us suppose we have a $2 \mathrm{DoF}$ system subjected to an external forcing $A_{0} \cos \left(\omega_{1} t+\phi\right)$ :

$$
\left\{\begin{array}{l}
m_{1} \ddot{x}+c_{1} \dot{x}+k_{1} x+m_{2} \ddot{y}=A_{0} \cos \left(\omega_{1} t+\phi\right) \\
m_{2} \ddot{y}+c_{2}(\dot{y}-\dot{x})+k_{2}(y-x)^{3}=0
\end{array}\right.
$$

This system is composed of a linear master system which is attached to a cubic NES. $m_{1}, c_{1}, k_{1}$ are system parameters of the master structure and $m_{2}, c_{2}$ and $k_{2}$ are system parameters of the NES. We introduce new parameters as it follows:

$\epsilon=\frac{m_{2}}{m_{1}}<<1, \omega^{2}=\frac{k_{1}}{m_{1}}, \zeta=\frac{c_{1}}{m_{1}}, \lambda=\frac{c_{2}}{m_{2}}, K=\frac{k_{2}}{m_{2}}$ and $A=\frac{A_{0}}{m_{1}}$. The Equation [1] can be re-written as:

$$
\left\{\begin{array}{l}
\ddot{x}+\zeta \dot{x}+\omega^{2} x+\epsilon \ddot{y}=A \cos \left(\omega_{1} t+\phi\right) \\
\ddot{y}+\lambda(\dot{y}-\dot{x})+K(y-x)^{3}=0
\end{array}\right.
$$

If we consider that the relative displacement $z$ is defined as $z=x-y$, then by reorganizing the system [2], one can get the following system:

$$
\left\{\begin{array}{l}
\ddot{x}+\zeta \dot{x}+\omega^{2} x+\epsilon(\ddot{x}-\ddot{z})=A \cos \left(\omega_{1} t+\phi\right) \\
\ddot{z}+\lambda \dot{z}+K z^{3}-\ddot{x}=0
\end{array}\right.
$$

$\omega$ is in fact the eigenfrequency of the master system and we assume that $\omega \approx \omega_{1}$, i.e. there is a 1:1 resonance between excitation and the master system.

\section{Overall assumptions and methodology}

As mentioned in the previous section, we force the system to be in a $1: 1$ resonance, i.e., $\omega \approx \omega_{1}$ which is the fundamental assumption of this paper. Let us suppose that the relative displacement $z$ is composed of several harmonics namely, $z_{O_{1}}, z_{O_{2}}, \ldots, z_{O_{N 1}}, z_{e_{1}}, z_{e_{2}}, \ldots, z_{O_{N} 2}, N_{1}+N_{2}=N$, which are associated with frequencies $o_{1} \omega_{1}, o_{2} \omega_{1}, \ldots, o_{N 1} \omega_{1}, e_{1} \omega_{1}, e_{2} \omega_{1} \ldots, e_{N 2} \omega_{1}$, respectively. We name this 
case as $\left(1: o_{1} \omega_{1}, o_{2} \omega_{1}, \ldots, o_{N 1} \omega_{1}, e_{1} \omega_{1}, e_{2} \omega_{1}, \ldots, e_{N 2} \omega_{1}\right)$ resonance, where $o$ and $e$ stand for the odd and the even numbers, respectively $\left(o \ldots \in\left\{1,3, \ldots, o_{N_{1}}\right\}\right.$ and $\left.e_{\ldots} \in\left\{0,2, \ldots, e_{N_{2}}\right\}\right)$. This is the same as the main resonance capture, i.e. $1: 1$ but by consideration of different harmonics. Then the relative displacement $z$ reads as:

$$
z=\sum_{j=1}^{N_{1}} z_{o_{j}}+\sum_{j=1}^{N_{2}} z_{e_{j}}
$$

Complex variables of Manevitch are introduced in order to shift the physical displacements of the system to the polar domain:

$$
\begin{cases}\varphi e^{i \omega_{1} t} & =\frac{\dot{x}}{\omega_{1}}+i x \\ \varphi_{o_{j}} e^{i\left(o_{j} \omega_{1}\right) t} & =\frac{\dot{z}_{o_{j}}}{o_{j} \omega_{1}}+i z_{o_{j}} \quad j \in\left\{1,2, \ldots, N_{1}\right\} \\ \varphi_{e_{j}} e^{i\left(e_{j} \omega_{1}\right) t} & =\frac{\dot{z}_{e_{j}}}{e_{j} \omega_{1}}+i z_{e_{j}} \quad j \in\left\{1,2, \ldots, N_{2}\right\}\end{cases}
$$

By applying above mentioned assumptions and change of variables to the system of [3], new set of complex form of first order differential equations is derived. Then, fixed points of the system can be revealed by imposing following criteria:

$$
\begin{cases}\dot{\varphi}=0 & \\ \dot{\varphi}_{o_{j}}=0 & j \in\left\{1,2, \ldots, N_{1}\right\} \\ \dot{\varphi}_{e_{j}}=0 & j \in\left\{1,2, \ldots, N_{2}\right\}\end{cases}
$$

So, as a summary the $2 \mathrm{DoF}$ system of equations is splitted to $N+1$ equations. The obtained set of equations can be solved by a step by step procedure which is introduced in following sections. It is important to mention that this kind of harmonic paring which is based on the splitting of the relative displacement, leads the system during its $1: 1$ resonance to different amplitudes and phases which are associated with different resonant frequencies; we call these as relative modes.

\section{1:1,3 Resonance}

Let us suppose following system parameters:

- parameters of the master substructure: $\omega=1, \zeta=0$;

- parameters of the NES: $\epsilon=0.005, K=488, \lambda=0.5$;

- parameters of the external excitation: $\omega_{1}=\omega=1, A=\epsilon \times 0.225$.

We set a criterion for neglecting some harmonics. In this example, all harmonics whose amplitudes are lower than $10 \%$ of the corresponding amplitude in the resonance are neglected. A numerical simulation was performed on the system of (3) by the 
RK45 function in Matlab with zero initial conditions to grasp the total behavior of the system. The frequency spectrum of the $x, y$ and $z$ are illustrated in Figures 13. Different harmonics of mentioned displacements are identified by the applying the Fourier transform on their response. The corresponding amplitude of the $y$ at the frequency $3 \omega_{1}$ is $\left|y\left(3 \omega_{1}\right)\right|=0.01383$, which is $6.1 \%$ of the amplitude at the frequency $\omega_{1}$, i.e. $\left|y\left(\omega_{1}\right)\right|=0.225$; so, according to our supposed criterion, harmonics $3,5 \ldots$ are negligible. If one compares the magnitudes of the relative displacement $z$ at different frequencies, the neglected harmonics will change: $\left|z\left(3 \omega_{1}\right)\right|=0.01391$ which is about $17.7 \%$ of $\left|z\left(\omega_{1}\right)\right|=0.07849$; as a result by using the idea of relative displacement, the third harmonic can not be neglected; see Figure 3. Therefore, for covering more accurate range of frequencies, we will seek for the resonance between $x$ and $z$.

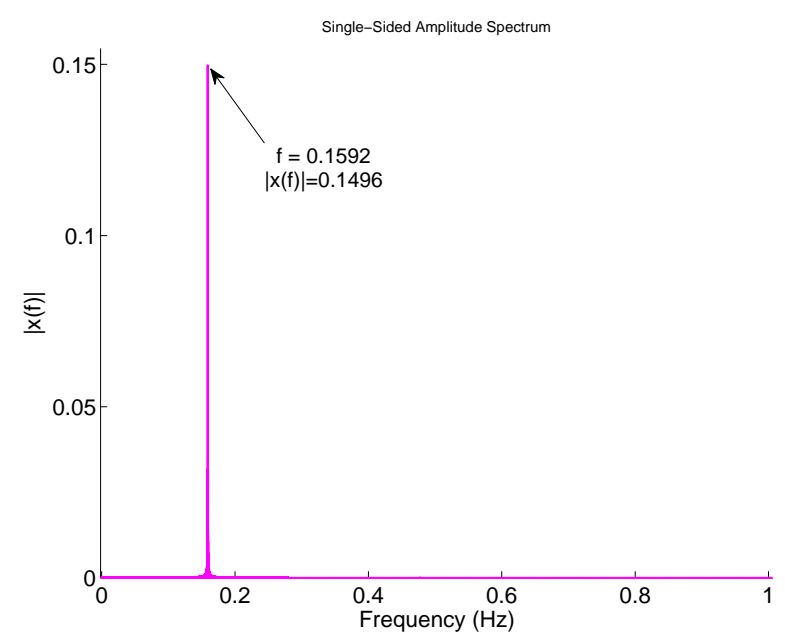

Figure 1. Frequency spectrum of $x$

\subsection{The asymptotic approach for the case of the 1:1,3 resonance}

In the above mentioned discussion, we pinpointed that two harmonics of the relative mode relevant to $\omega_{1}$ and $3 \omega_{1}$ frequencies are significant; we call this as the 1:1,3 resonance. We will deal with this problem by endowing splitting harmonics and the asymptotic approaches. Let us suppose that the relative displacements $z$ can be decomposed as it follows:

$$
z=z_{1}+z_{3}
$$


130 EJCM - 20/2011. Dynamics of materials, structures and systems

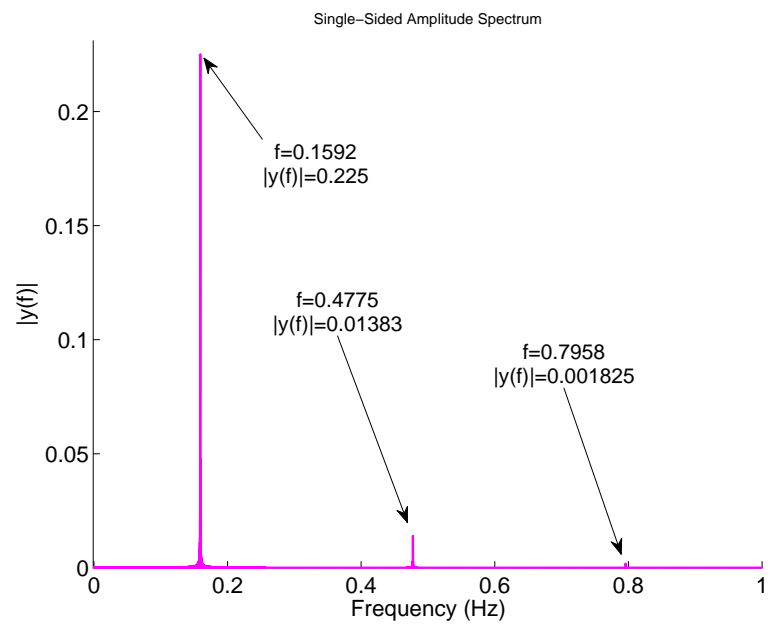

Figure 2. Frequency spectrum of $y$

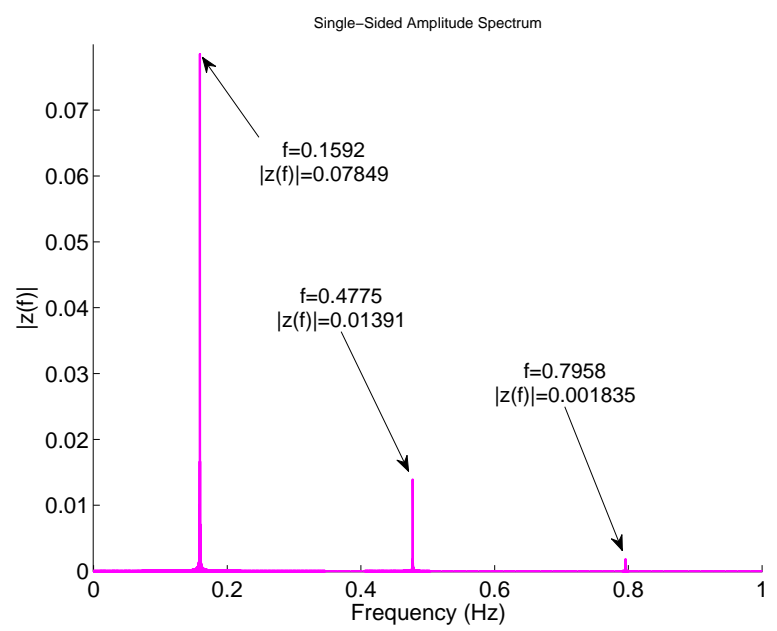

Figure 3. Frequency spectrum of $z$

where $z_{1}$ and $z_{3}$ are those parts of the relative displacement relevant to the resonance frequency $\omega_{1}$ and harmonic frequency $3 \omega_{1}$, respectively. This assumption will lead [3] to the following system of equations:

$$
\begin{cases}\ddot{x}+\zeta \dot{x}+\omega^{2} x+\epsilon\left(\ddot{x}-\ddot{z}_{1}-\ddot{z}_{3}\right) & =A \cos \left(\omega_{1} t+\phi\right) \\ \ddot{z}_{1}+\ddot{z}_{3}+\lambda\left(\dot{z}_{1}+\dot{z}_{3}\right)+K\left(z_{1}+z_{3}\right)^{3} & =\ddot{x}\end{cases}
$$


In order to deal with the nonlinear modes, following Manevitch's complex function of variables are introduced to the system [8]:

$$
\begin{cases}\varphi e^{i \omega_{1} t} & =\frac{\dot{x}}{\omega_{1}}+i x \\ \varphi_{1} e^{i \omega_{1} t} & =\frac{\dot{z}_{1}}{\omega_{1}}+i z_{1} \\ \varphi_{3} e^{i 3 \omega_{1} t} & =\frac{\dot{z}_{3}}{3 \omega_{1}}+i z_{3}\end{cases}
$$

Then, associated equations to $\omega_{1}$ and $3 \omega_{1}$ are summarized in the Equation [10] and the Equation [11], respectively.

$$
\begin{aligned}
& \left\{\begin{array}{r}
2(1+\epsilon) \omega_{1} \dot{\varphi}-2 \epsilon \omega_{1} \dot{\varphi}_{1}+i\left[(1+\epsilon) \omega_{1}^{2}-\omega^{2}\right] \varphi+ \\
\zeta \omega_{1} \varphi-i \epsilon \omega_{1}^{2} \varphi_{1}
\end{array}=\underline{A}\right. \\
& 24 \omega_{1} \dot{\varphi}_{3}+\left(12 \lambda \omega_{1}+36 i \omega_{1}^{2}-6 i K\left|\varphi_{1}\right|^{2}-3 i K\left|\varphi_{3}\right|^{2}\right) \varphi_{3}=-i K \varphi_{1}^{3}
\end{aligned}
$$

The steady state of the system can be evaluated by following system of equations:

$$
\left\{\begin{array}{l}
\dot{\varphi}=0 \\
\dot{\varphi}_{1}=0 \\
\dot{\varphi}_{3}=0
\end{array}\right.
$$

Now, relevant equations to $\omega_{1}$ and $3 \omega_{1}$ are collected in Equations [13] and [14], respectively.

$$
\left\{\begin{array}{l}
i\left[(1+\epsilon) \omega_{1}^{2}-\omega^{2}\right] \varphi+\zeta \omega_{1} \varphi- \\
i \epsilon \omega_{1}^{2} \varphi_{1}=\underline{A} \\
4 \lambda \omega_{1} \varphi_{1}+4 i \omega_{1}^{2} \varphi_{1}-3 i K \varphi_{1}\left|\varphi_{1}\right|^{2}-6 i K \varphi_{1}\left|\varphi_{3}\right|^{2}+ \\
3 i K \bar{\varphi}_{1}^{2} \varphi_{3}=4 i \omega_{1}^{2} \varphi
\end{array}\right.
$$

where $\underline{A}=A e^{i \phi}$.

$$
\left(12 \lambda \omega_{1}+36 i \omega_{1}^{2}-6 i K\left|\varphi_{1}\right|^{2}-3 i K\left|\varphi_{3}\right|^{2}\right) \varphi_{3}=-i K \varphi_{1}^{3}
$$

In the following subsection a novel iterative technique will be instructed in order to deal with the obtained equations.

\subsection{An iterative technique for evaluating the absolute values of harmonics}

Let us suppose the 1:1 resonance where the relevant harmonic to the $3 \omega_{1}$ is small, i.e., $\left|\varphi_{3}\right|$ is much more smaller than the $\left|\varphi_{1}\right|$, so as a rough estimation we neglect the 
effect of the $\varphi_{3}$ on $\varphi_{1}$ in the second equation of the system [13]. This will lead us to determining the $\varphi_{1}$ without knowing the $\varphi_{3}$ which is the case of the $1: 1$ resonance. Now, according to the Equation [14] we will have:

$$
\varphi_{3}=-\frac{i K \varphi_{1}^{3}}{12 \lambda \omega_{1}+36 i \omega_{1}^{2}-6 i K\left|\varphi_{1}\right|^{2}-3 i K\left|\varphi_{3}\right|^{2}}
$$

Now, we suppose that parameters of the NES i.e. $(K, \lambda)$ vary slightly while other parameters are fixed. We define a new parameter $C=2\left|\varphi_{1}\right|^{2}+\left|\varphi_{3}\right|^{2}$ which varies slightly. By considering this assumption on Equation [15] we will have:

$$
\begin{aligned}
\left|\varphi_{3}\right| & =\left|\frac{-i K \varphi_{1}^{3}}{12 \lambda \omega_{1}+36 i \omega_{1}^{2}-3 i K C}\right| \\
& =\frac{1}{3} \frac{K\left|\varphi_{1}\right|^{3}}{\sqrt{\left(4 \lambda \omega_{1}\right)^{2}+\left(12 \omega_{1}^{2}-K C\right)^{2}}}
\end{aligned}
$$

From the first equation of the system [13], one can reach to the following equation:

$$
\begin{aligned}
\varphi & =\frac{\underline{A}+i \epsilon \omega_{1}^{2} \varphi_{1}}{i\left[(1+\epsilon) \omega_{1}^{2}-\omega^{2}\right]+\zeta \omega_{1}} \\
& =\alpha_{0}+\alpha_{1} \varphi_{1},
\end{aligned}
$$

where $\alpha_{0}=\frac{\underline{A}}{i\left[(1+\epsilon) \omega_{1}^{2}-\omega^{2}\right]+\zeta \omega_{1}}, \alpha_{1}=\frac{i \epsilon \omega_{1}^{2}}{i\left[(1+\epsilon) \omega_{1}^{2}-\omega^{2}\right]+\zeta \omega_{1}}$. By substituting $\varphi$ in the second equation of the system [13] we will have:

$$
\begin{aligned}
& 4 \lambda \omega_{1} \varphi_{1}+4 i \omega_{1}^{2} \varphi_{1}-3 i K \varphi_{1}\left|\varphi_{1}\right|^{2}-6 i K \varphi_{1}\left|\varphi_{3}\right|^{2}+ \\
& 3 i K \bar{\varphi}_{1}^{2} \varphi_{3}=4 i \omega_{1}^{2}\left(\alpha_{0}+\alpha_{1} \varphi_{1}\right)
\end{aligned}
$$

By considering Equation [15] just in the term $3 i K \bar{\varphi}_{1}^{2} \varphi_{3}$ of the Equation [18], following system can be obtained:

$$
\begin{aligned}
& \varphi_{1}\left[4 \lambda \omega_{1}+4 i \omega_{1}^{2}\left(1-\alpha_{1}\right)-3 i K\left(\left|\varphi_{1}\right|^{2}+2\left|\varphi_{3}\right|^{2}\right)+\right. \\
& \left.\frac{K^{2}\left|\varphi_{1}\right|^{2}}{4 \lambda \omega_{1}+12 i \omega_{1}^{2}-i K\left(2\left|\varphi_{1}\right|^{2}+\left|\varphi_{3}\right|^{2}\right)}\right]=4 i \omega_{1}^{2} \alpha_{0}
\end{aligned}
$$

Now, we have a system which can be defined by Equations [14] and [19]. By defining following variables:

$$
\begin{gathered}
\left|\varphi_{j}\right|=N_{j}, \quad j=1,3 \\
Z_{j}=N_{j}^{2}, \quad j=1,3
\end{gathered}
$$

one can reach to the following general form of system of equations:

$$
F_{k}\left(Z_{1}, Z_{3}\right)=0, \quad k=1,2 \text {. }
$$


$F_{1}$ and $F_{2}$ can be evaluated by endowing Equations [19] and [14], respectively.

$$
\begin{aligned}
& F_{1}=a_{1} Z_{1}+a_{2} Z_{1}^{2}+a_{3} Z_{1}^{3}+a_{4} Z_{1}^{4}+a_{5} Z_{1}^{5}-a_{0}=0 \\
& F_{2}=b_{1} Z_{3}+b_{2} Z_{3}^{2}+b_{3} Z_{3}^{3}-b_{0}=0
\end{aligned}
$$

The expression of variables of the Equations [23] and [24] are defined as:

$$
\begin{aligned}
& a_{0}=256 \omega_{1}^{6}\left|\alpha_{0}\right|^{2} \lambda^{2}+2304 \omega_{1}^{8}\left|\alpha_{0}\right|^{2}+16 \omega_{1}^{4}\left|\alpha_{0}\right|^{2} K^{2} Z_{3}^{2}-384 \omega_{1}^{6}\left|\alpha_{0}\right|^{2} K Z_{3} \\
& a_{1}=256 \lambda^{4} \omega_{1}^{4}+2560 \lambda^{2} \omega_{1}^{6}+2304 \omega_{1}^{8} \alpha_{11}^{2}-4608 \omega_{1}^{8} \alpha_{11}+2304 \omega_{1}^{8} \alpha_{12}^{2}- \\
& 912 \omega_{1}^{2} K^{3} Z_{3}^{3}-64 \omega_{1}^{4}\left|\alpha_{0}\right|^{2} K^{2} Z_{3}-7296 \omega_{1}^{6} K Z_{3}+6352 \omega_{1}^{4} K^{2} Z_{3}^{2}+ \\
& 768 \omega_{1}^{6}\left|\alpha_{0}\right|^{2} K-512 \lambda^{2} \omega_{1}^{6} \alpha_{11}+512 \lambda^{3} \omega_{1}^{5} \alpha_{12}+4608 \omega_{1}^{7} \alpha_{12} \lambda+36 K^{4} Z_{3}^{4}+ \\
& 256 \omega_{1}^{6} \alpha_{11}^{2} \lambda^{2}+32 \omega_{1}^{3} \alpha_{12} \lambda K^{2} Z_{3}^{2}+768 \lambda^{2} \omega_{1}^{4} \alpha_{11} K Z_{3}-768 \omega_{1}^{5} \alpha_{12} \lambda K Z_{3} \\
& +2304 \omega_{1}^{8}+256 \omega_{1}^{6} \alpha_{12}^{2} \lambda^{2}-1184 \omega_{1}^{4} \alpha_{11} K^{2} Z_{3}^{2}+592 \lambda^{2} \omega_{1}^{2} K^{2} Z_{3}^{2}+ \\
& 48 K^{3} Z_{3}^{3} \omega_{1}^{2} \alpha_{11}-384 \omega_{1}^{6} \alpha_{11}^{2} K Z_{3}-1152 \lambda^{2} \omega_{1}^{4} K Z_{3} \\
& +16 \omega_{1}^{4} \alpha_{11}^{2} K^{2} Z_{3}^{2}+7680 \omega_{1}^{6} \alpha_{11} K Z_{3}-384 \omega_{1}^{6} \alpha_{12}^{2} K Z_{3}+16 \omega_{1}^{4} \alpha_{12}^{2} K^{2} Z_{3}^{2} \\
& a_{2}=640 \lambda^{2} \omega_{1}^{2} K^{2} Z_{3}+64 \omega_{1}^{4} \alpha_{11}^{2} K^{2} Z_{3}-768 \omega_{1}^{6} \alpha_{11}^{2} K-3008 \omega_{1}^{4} \alpha_{11} K^{2} Z_{3}- \\
& 2808 \omega_{1}^{2} K^{3} Z_{3}^{2}+384 \lambda^{2} \omega_{1}^{4} \alpha_{11} K+64 \omega_{1}^{4} \alpha_{12}^{2} K^{2} Z_{3}-4224 \omega_{1}^{6} K- \\
& 64 \omega_{1}^{4}\left|\alpha_{0}\right|^{2} K^{2}-1152 \lambda^{2} \omega_{1}^{4} K-1536 \omega_{1}^{5} \alpha_{12} \lambda K+4992 \omega_{1}^{6} \alpha_{11} K- \\
& 768 \omega_{1}^{6} \alpha_{12}^{2} K+180 K^{4} Z_{3}^{3} 64 \omega_{1}^{4}\left|\alpha_{0}\right|^{2} K^{2}+128 \omega_{1}^{3} \alpha_{12} \lambda K^{2} Z_{3}+ \\
& 216 K^{3} Z_{3}^{2} \omega_{1}^{2} \alpha_{11}+8128 \omega_{1}^{4} K^{2} Z_{3} \\
& a_{3}=64 \omega_{1}^{4} \alpha_{12}^{2} K^{2}+285 K^{4} Z_{3}^{2}+160 \omega_{1}^{3} \alpha_{12} \lambda K^{2}+280 K^{3} \omega_{1}^{2} \alpha_{11} Z_{3}- \\
& 1184 \omega_{1}^{4} \alpha_{11} K^{2}+64 \omega_{1}^{4} \alpha_{11}^{2} K^{2}-2080 \omega_{1}^{2} K^{3} Z_{3}+2416 \omega_{1}^{4} K^{2}+240 \lambda^{2} \omega_{1}^{2} K^{2} \\
& a_{4}=80 K^{3} \omega_{1}^{2} \alpha_{11}-440 \omega_{1}^{2} K^{3}+150 K^{4} Z_{3} \\
& a_{5}=25 K^{4} \\
& b_{0}=Z_{1}^{3} \\
& b_{1}=9 \\
& b_{2}=\frac{36 K Z_{1}-216 \omega_{1}^{2}}{K} \\
& b_{3}=\frac{36 K^{2} Z_{1}^{2}-432 \omega_{1}^{2} K Z_{1}+144 \lambda^{2} \omega_{1}^{2}+1296 \omega_{1}^{4}}{K^{2}}
\end{aligned}
$$

where $\alpha_{11}=\Re\left(\alpha_{1}\right), \alpha_{12}=\Im\left(\alpha_{1}\right)$. Since the influence of $Z_{3}$ on $Z_{1}$ is very small, so we start from Equation [23] by assuming the initial value $Z_{3}=Z_{3, \text { start }}=0$ and we obtain the value of $Z_{1}$ which we name it $Z_{1,0}$. By substituting $Z_{1,0}$ in [24] we will be able to evaluate $Z_{3}$, which we call it $Z_{3,0}$. The procedure will be repeated at each step $j$ in order to evaluate $Z_{1, j}, Z_{3, j}$. The stopping criteria for the iterative technique is $\left|F_{k}\left(Z_{1}, Z_{3}\right)\right|<r_{F}$, where $r_{F}$ is a very small positive number. The procedure is illustrated schematically on Table 1 . 
Table 1. The iterative procedure

\begin{tabular}{|c|c|c|c|c|c|c|}
\hline $7-0$ & $\begin{array}{c}Z_{1,0} \\
\downarrow\end{array}$ & ... & $\begin{array}{c}Z_{1, k} \\
\downarrow\end{array}$ & $\begin{array}{c}Z_{1, k+1} \\
\downarrow\end{array}$ & $\ldots$ & $\begin{array}{c}Z_{1, n} \\
\downarrow\end{array}$ \\
\hline$Z_{3, \text { start }}=0$ & $Z_{3,0}$ & $\cdots$ & $Z_{3, k}$ & $Z_{3, k+1}$ & $\ldots$ & $Z_{3, n}$ \\
\hline
\end{tabular}

\subsubsection{Two examples}

As a first example let us take values of the example which are given in Section 4. Numerical and analytical results of the chosen system which are obtained by the RK45 function in Matlab and the iterative technique, respectively are illustrated in Table 2. This table shows a good agreement between numerical and iterative results (see Figure 3 as well). For the second example we just change parameters of the NES as it follows:

$K=900, \lambda=1$. Numerical results for the $y$ and $z$ are illustrated in Figure 4 and 5, respectively. From these figures, $\frac{\left|y_{3}\right|}{\left|y_{1}\right|}=5.7 \%$ and $\frac{\left|z_{3}\right|}{\left|z_{1}\right|}=20.7 \%$ which proves again the importance of considering the relative mode instead of the absolute one in evaluating the better system parameters.

Table 2. Iterative and numerical results: first example

\begin{tabular}{|c|c|c|}
\hline Amplitudes of harmonics & $N_{1}$ & $N_{3}$ \\
\hline Iterative result & 0.07912 & 0.01311 \\
\hline Numerical result & 0.07849 & 0.01391 \\
\hline
\end{tabular}

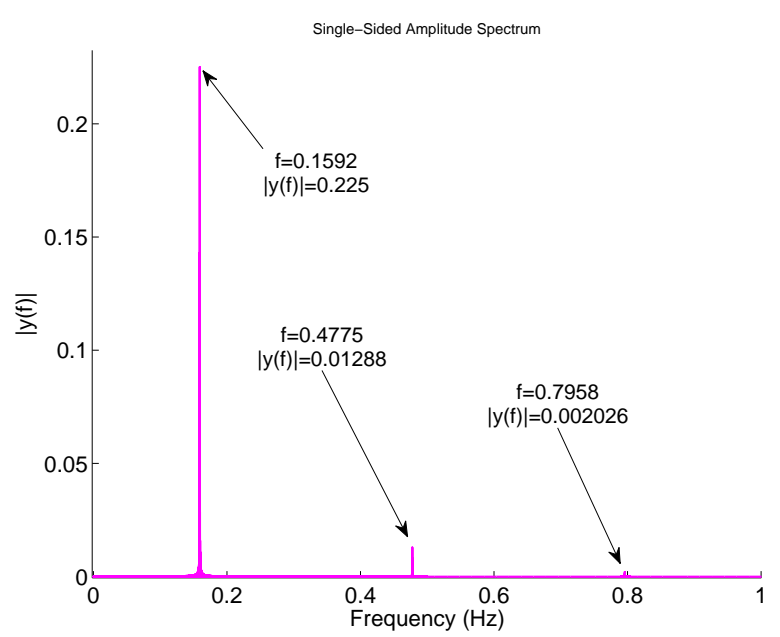

Figure 4. Frequency spectrum of $y$

Comparison of the iterative and numerical results are gathered in Table 3 which depicts a good agreement between iterative and numerical results. 


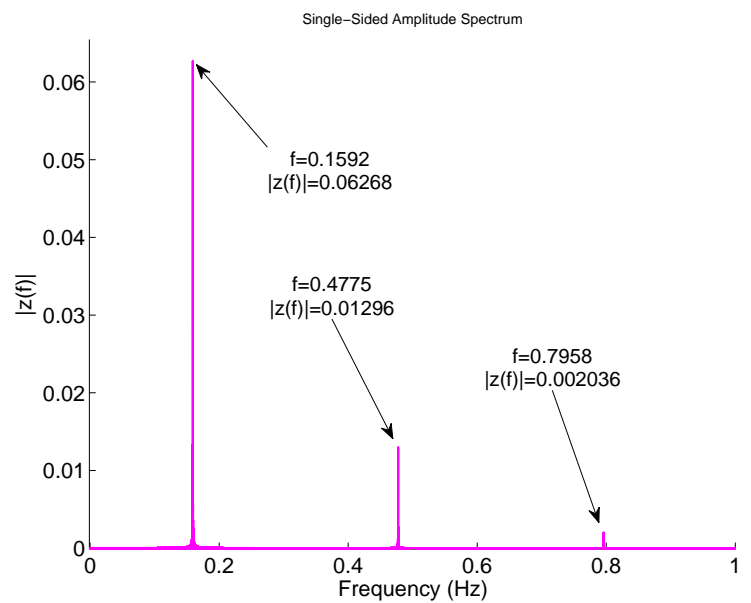

Figure 5. Frequency spectrum of $z$

Table 3. Iterative and numerical results: second example

\begin{tabular}{|c|c|c|}
\hline Amplitudes of harmonics & $N_{1}$ & $N_{3}$ \\
\hline Iterative result & 0.06319 & 0.01230 \\
\hline Numerical result & 0.06268 & 0.01209 \\
\hline
\end{tabular}

\section{A complete iterative technique}

\subsection{Definition of the complete iterative technique}

The iterative procedure which was introduced in the previous section deals with the real and positive valued unknowns, i.e. $\left|\varphi_{1}\right|,\left|\varphi_{3}\right|$; the main advantage of this method is its fast convergence. In the general case, the dynamical system will have more than two unknowns and they are complex. To this end, a general form of the iterative technique is introduced in order to deal with more general cases with any kinds of system unknowns. The first equation of the system [13] is invariant for all type of localizations of the relative mode, so as a result the Equation [17] will be invariant too. By collecting Equations [18] and [14], one can reach to the following system:

$$
\left\{\begin{array}{l}
{\left[4 \lambda \omega_{1}+4 i \omega_{1}^{2}\left(1-\alpha_{1}\right)-3 i K\left|\varphi_{1}\right|^{2}-\right.} \\
\left.6 i K\left|\varphi_{3}\right|^{2}\right] \varphi_{1}=4 i \omega_{1}^{2} \alpha_{0}-3 i K \bar{\varphi}_{1}^{2} \varphi_{3} \\
\left(12 \lambda \omega_{1}+36 i \omega_{1}^{2}-3 i K\left|\varphi_{3}\right|^{2}-6 i K\left|\varphi_{1}\right|^{2}\right) \varphi_{3}=-i K \varphi_{1}^{3}
\end{array}\right.
$$

Let us suppose that:

$$
\varphi_{j}=N_{j} e^{i \delta_{j}}, j=1,3
$$


By considering the introduced variable in Equation [21], the system of [25] in the following compact form will be as:

$$
G_{j}\left(Z_{j}\right)=\left|A_{j}\right|, \quad j=1,3
$$

where $G_{j}(x)=\sum_{k=1}^{3} a_{j, k} x^{k}, a_{j, k}=a_{j, k}\left(Z_{l}\right), \quad l \neq j$ and $A_{1}=4 i \omega_{1}^{2} \alpha_{0}-3 i K \bar{\varphi}_{1}^{2} \varphi_{3}$, $A_{3}=-i K \varphi_{1}^{3} . G_{j}\left(Z_{j}\right)$ in the system of [27] is a polynomial which its unknowns are norms of complex amplitudes. Phases of harmonics can be evaluated by considering the system [25] as it follows:

$$
\left\{\begin{aligned}
e^{i \delta_{1}} & =\frac{A_{1}}{\left(\lambda \omega_{1}+4 i \omega_{1}^{2}\left(1-\alpha_{1}\right)-3 i K N_{1}^{2}-6 i K N_{3}^{2}\right) N_{1}} \\
e^{i \delta_{3}} & =\frac{A_{3}}{\left(12 \lambda \omega_{1}+36 i \omega_{1}^{2}-3 i K N_{3}^{2}-6 i K N_{1}^{2}\right) N_{3}}
\end{aligned}\right.
$$

Now, the overall system can be solved by the algorithm which is depicted in Figure 6. In the introduced algorithm, $Z_{1 t}, Z_{3 t}, \delta_{1 t}$ and $\delta_{3 t}$ are values of the $Z_{1}, Z_{3}, \delta_{1}$ and $\delta_{3}$ at the previous step, while $Z_{1 s}, Z_{3 s}, \delta_{1 s}$ and $\delta_{3 s}$ are values of $Z_{1}, Z_{3}, \delta_{1}$ and $\delta_{3}$ at the current step of iteration.

\subsection{The full frequency response of the system with the assumption of the 1:1,3 resonance: a case study}

We are interested to grasp the evolution of the response of the system by sweeping the resonance frequency in an interval. Let us consider the example of the Section 4 and with the new NES parameters: $K=25$ and $\lambda=0.5$. We consider that $\omega_{1}=$ $\omega+\epsilon \sigma$ where the detuning parameter $\sigma$ sweeps the interval $[-0.72,6]$. Maximum iterative and numerical responses of the system for each frequency in the stationary regime which are obtained by the iteration technique and the RK45 function of the MATLAB package are illustrated in Figures 7- 9. Overall views of these diagrams show an acceptable agreement between numerical and iterative results, but there are some zones which the numerical and iterative results are not in good agreements. These disagreements indicate the possibility of existence of other kinds of behaviors which are not considered here and they demand further studies.

\section{Other type of localization: $1: 1,3,0,2$ resonance}

Let us consider a NES with the $K=511$ and $\lambda=0.5$. The response of the system for the relative displacement which is obtained by the numerical simulation in MATLAB with the RK45 function is depicted in Figure 10. This figure shows that there are other harmonics which should be considered, such as 1:1,3,0,2; we call these 


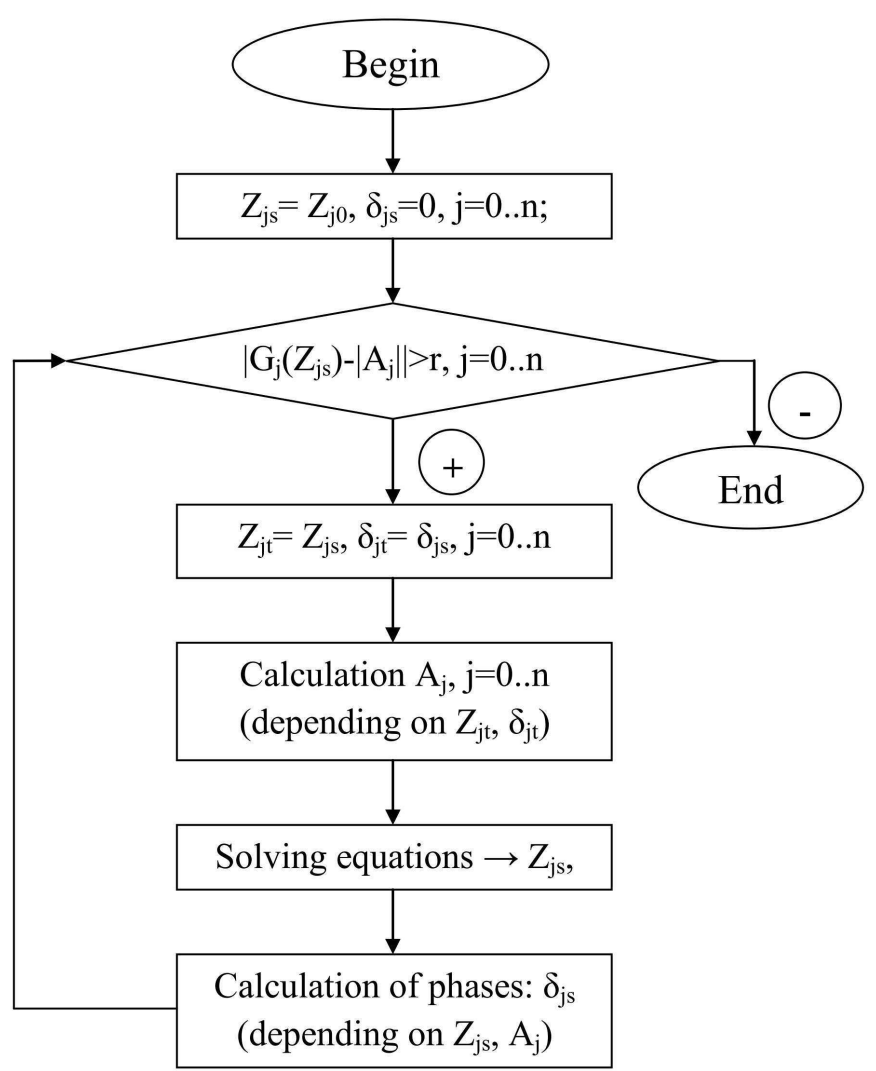

Figure 6. The algorithm of the iterative technique

kinds of harmonics in the form of successive odd and even resonance cases such as $1: 1,3, \ldots, 0,2 \ldots$ In the following sub-section we will deal with these general case of localization.

\subsection{An asymptotic approach}

By implementing the general approach of Section 3, complex variables of the system [5] build a set of equations which in a compact form reads as:

$$
G_{n_{j}}\left(\left|\varphi_{n_{j}}\right|^{2}\right)=\left|A_{n_{j}}\right|^{2}, \quad n_{j} \in\left\{o_{1}, o_{2}, \ldots, o_{N 1}, e_{1}, e_{2}, \ldots, e_{N 2}\right\}
$$

This system is similar to the system of [27]. It is worthwhile to mention that in applying complex variables to system unknowns, the harmonic $z_{0}$ which is associated with the zero frequency, will read as $z_{0}=\varphi_{0}$, because it is a constant. The general form 


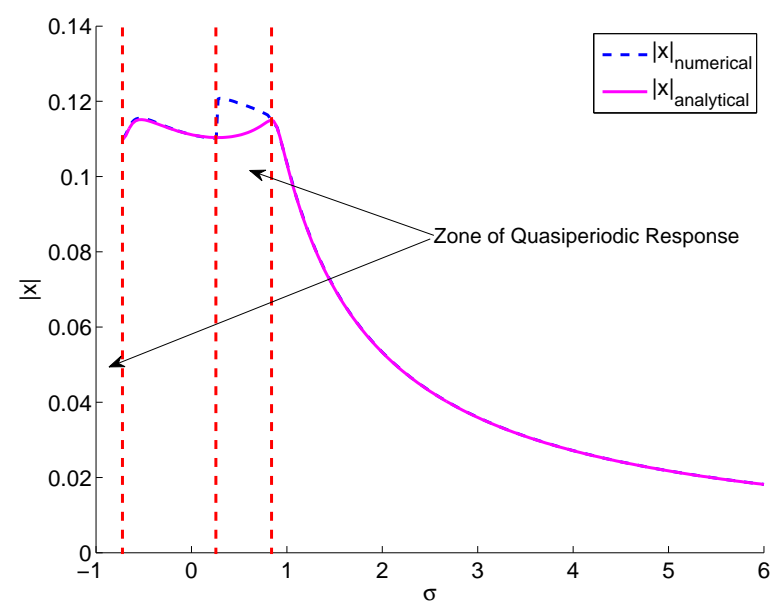

Figure 7. $|x|$ vs. $\sigma$ in the 1:1,3 resonance

of the iteration technique which is introduced in Section 5 can be endowed in order to solve the above mentioned system of equations but this case will generate higher order of equations with more unknowns.

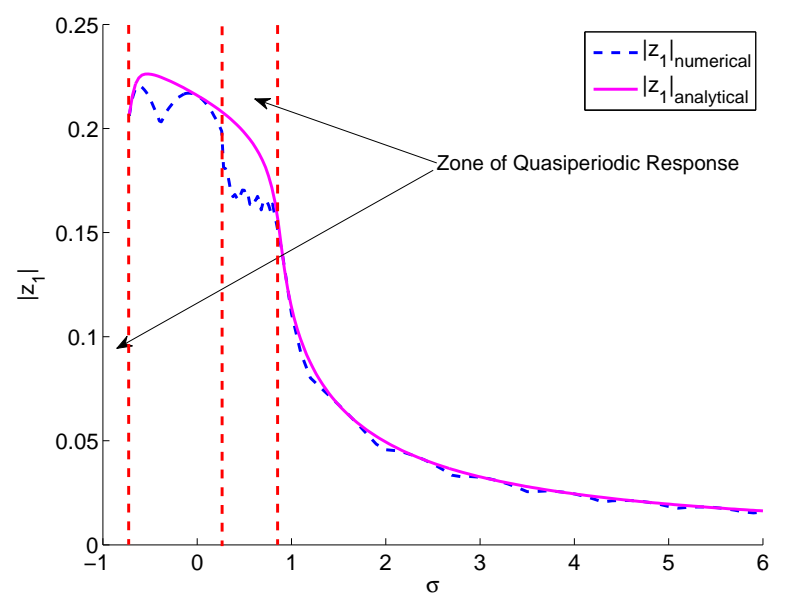

Figure 8. $\left|z_{1}\right|$ vs. $\sigma$ in the $1: 1,3$ resonance 


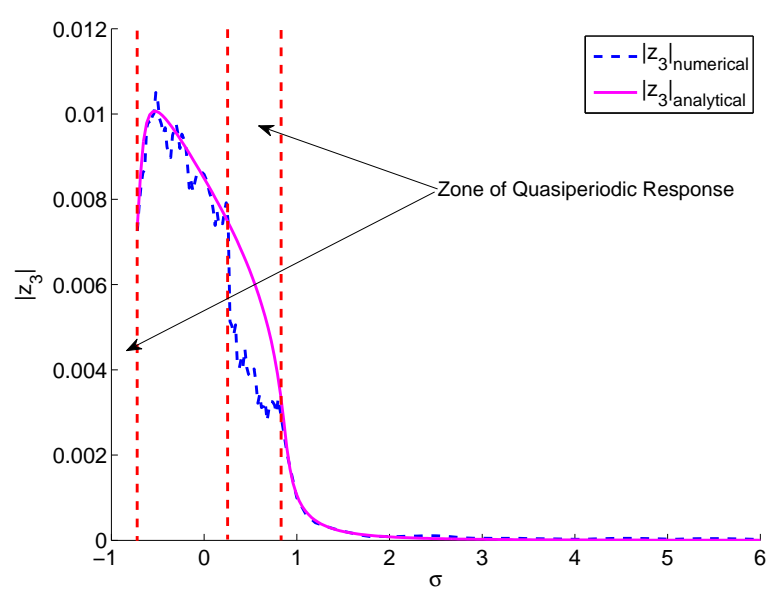

Figure 9. $\left|z_{3}\right|$ vs. $\sigma$ in the 1:1,3 resonance

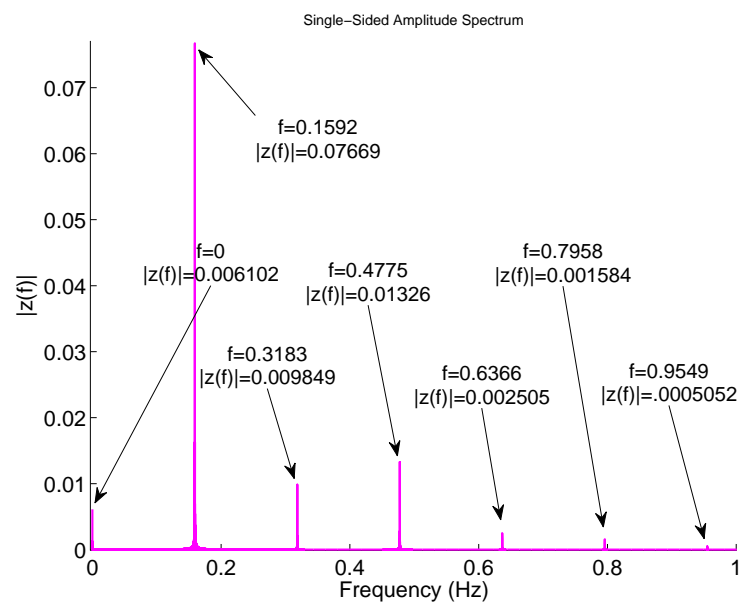

Figure 10. Frequency spectrum of $z$

\subsection{The importance of correct resonance paring: a case study}

Considering the correct resonance paring is an important issue in localization. Let us consider 1:1,3,5,6,7,9 resonance case. Iterative results are collected in Table 4. Obtained result for the relevant harmonic of the $9 \omega_{1}\left(\left|z_{9}\right|\right)$ is much more smaller than the harmonic of the central frequency, i.e., $\left|z_{1}\right|$; so we didn't consider more harmonics in 
this case study. We were not able to obtain the same results by "numerical" procedure which means that we looked for solution corresponding to $1: 1,3,5,6,7,9$ resonance case that does not exist (this assumption is not correct).

Table 4. Iterative results for the 1:1,3,5,6,7,9 resonance

\begin{tabular}{|c|c|c|c|c|c|}
\hline Amplitudes & $N_{1}$ & $N_{3}$ & $N_{5}$ & $N_{7}$ & $N_{9}$ \\
\hline Results & 0.093 & 0.036 & 0.0078 & 0.0009 & 0.00006 \\
\hline
\end{tabular}

Now, let us consider the 1:1,3,0,2 resonance; so $N=4$ in Equation [4]. We will obtain following system of equations for amplitudes:

$$
\left\{\begin{array}{l}
8 z_{0}^{3}+12\left(\left|\varphi_{1}\right|^{2}+\left|\varphi_{2}\right|^{2}+\left|\varphi_{3}\right|^{2}\right) z_{0}=A_{0} \\
\varphi_{1}\left(4 i \omega_{1}^{2} \alpha_{11}+12 i K z_{0}^{2}+6 i K\left|\varphi_{3}\right|^{2}+6 i K\left|\varphi_{2}\right|^{2}-\right. \\
\left.4 \omega_{1}^{2} \alpha_{12}-4 i \omega_{1}^{2}-4 \lambda \omega_{1}+3 i K\left|\varphi_{1}\right|^{2}\right)=A_{1} \\
\left(6 i K\left|\varphi_{3}\right|^{2}+6 i K\left|\varphi_{1}\right|^{2}+12 i K z_{0}^{2}+3 i K\left|\varphi_{2}\right|^{2}-\right. \\
\left.8 \lambda \omega_{1}-16 i \omega_{1}^{2}\right) \varphi_{2}=A_{2} \\
3\left(2 i K\left|\varphi_{1}\right|^{2}+4 i K z_{0}^{2}-12 i \omega_{1}^{2}+2 i K\left|\varphi_{2}\right|^{2}+i K\left|\varphi_{3}\right|^{2}-\right. \\
\left.4 \lambda \omega_{1}\right) \varphi_{3}=A_{3}
\end{array}\right.
$$

where:

$$
\left\{\begin{array}{l}
A_{0}=6 i \varphi_{1} \varphi_{2} \bar{\varphi}_{3}-3 i \bar{\varphi}_{1}^{2} \varphi_{2}+3 i \varphi_{1}^{2} \bar{\varphi}_{2}-6 i \bar{\varphi}_{1} \bar{\varphi}_{2} \varphi_{3} \\
A_{1}=12 K z_{0} \bar{\varphi}_{2} \varphi_{3}+3 i K \bar{\varphi}_{1}^{2} \varphi_{3}-3 i K \varphi_{2}^{2} \bar{\varphi}_{3}+12 K z_{0} \bar{\varphi}_{1} \varphi_{2}-4 i \omega_{1}^{2} \alpha_{0} \\
A_{2}=12 K z_{0} \bar{\varphi}_{1} \varphi_{3}-6 K z_{0} \varphi_{1}^{2}-6 i K \varphi_{1} \bar{\varphi}_{2} \varphi_{3} \\
A_{3}=K\left(i \varphi_{1}^{3}-12 z_{0} \varphi_{1} \varphi_{2}-3 i \bar{\varphi}_{1} \varphi_{2}^{2}\right)
\end{array}\right.
$$

In the first equation of the system [31], $A_{0}$ is a real valued number. If $\left|\varphi_{2}\right|=0$ then $A_{0}=0$; this means that at the first equation of the system [30], $z_{0}=0$. So, we can conclude that existence of the relevant harmonic of the frequency $2 \omega_{1}$ is necessary in order to we have non-zero $z_{0}$. The importance of the existence of $z_{0}$ will be more highlighted by looking at the relevant equation for evaluating $A_{2}$ at the system [31]. This gives us a good hint about choosing a good non-zero initial values for $z_{0}$ and $Z_{2}$ in order to avoid disturbing the fundamental localization assumption in the 1:1,3,0,2 resonance. For our example, we consider $z_{0 s}=-10^{-3}$ and $Z_{2 s}=10^{-3}$ as the starting values for $z_{0}$ and $Z_{2}$ in the proposed iteration technique. By endowing the 1:1,3,0,2 assumption for the resonance we are able to match numerical and iterative results, which means the more realistic assumption for the localization. Results are collected in Table 5. The given example illustrates the importance of choosing more harmonics which present more realistic behavior of the system under consideration. Although there are some errors between numerical and iterative results-maximum $10 \%$ of the 
error between numerical and iterative results with respect to the numerical results of the resonant harmonic $N_{1}$ for the harmonic $N_{3}$ which has very small amplitude, i.e. $\frac{|0.013-0.0212993|}{|0.076|}=10 \%$-, but it reveals the capability of the proposed iterative technique in detecting the more realistic system behavior by choosing right resonance pairs.

Table 5. Iterative and numerical results for the 1:1,3,0,2 resonance

\begin{tabular}{|c|c|c|c|c|}
\hline Amplitude & $z_{0}$ & $N_{1}$ & $N_{2}$ & $N_{3}$ \\
\hline Numerical results & -0.0061 & 0.076 & 0.0098 & 0.013 \\
\hline Iterative results & -0.00307109 & 0.0748807 & 0.0103894 & 0.0212993 \\
\hline
\end{tabular}

\section{Conclusion}

The importance of the "relative" displacement in a compound 2DoF nonlinear system which is composed of a master linear DoF and a slave cubic nonlinear DoF was highlighted. We showed that by endowing the idea of the relative displacement we will be able to consider more harmonics than the case of the absolute displacement. A fast convergent and then a robust "interactive" techniques were proposed in order to have a deep envision about the system for assumed different resonance paring. Thanks to the proposed iterative technique, we were able to reveal the importance of choosing right resonance paring and localization in the compound system. Further studies are going on in order to find different resonance boundaries.

\section{Acknowledgements}

This work has been supported by "Agence Nationale de la Recherche" (ANR) in the frame of the project ADYNO ANR-07-BLAN-0193.

\section{References}

Gendelman O. V., Starosvetsky Y., " Quasiperiodic response regimes of linear oscillator coupled to nonlinear energy sink under periodic forcing”, Journal of Applied Mechanics, vol. 74, $\mathrm{n}^{\circ} 2$, p. 325-331, 2007.

Jiang X., McFarland M., Bergman L. A., Vakakis A. F., " Steady state passive nonlinear energy pumping in coupled oscillators: theoretical and experimental results", Nonlinear Dynamics, vol. 33, nº 1, p. 87-102, 2003.

Pham T. T., Pernot S., Lamarque C.-H., " Passive control of one degree of freedom nonlinear quadratic oscillator under parametric resonance", Communications in Nonlinear Science and Numerical Simulation, vol. 16, n 5, p. 2279-2288, 2011.

Schmidt F., Lamarque C.-H., “ Energy pumping for mechanical systems involving non-smooth saint-venant terms", International Journal of Non-Linear Mechanics, vol. 45, n 9, p. 866$875,2010$. 
142 EJCM - 20/2011. Dynamics of materials, structures and systems

Silva C. W. D., Vibration and shock handbook, CRC Press, U.S., 2005.

Starosvetsky Y., Gendelman O. V., " Response regimes of linear oscillator coupled to nonlinear energy sink with harmonic forcing and frequency detuning", Journal of Sound and Vibration, vol. 315, $\mathrm{n}^{\circ}$ 3, p. 746-765, 2008a.

Starosvetsky Y., Gendelman O. V., " Strongly modulated response in forced 2dof oscillatory system with essential mass and potential asymmetry", Physica D, vol. 237, n ${ }^{\circ} 13$, p. 1719$1733,2008 b$.

Vakakis A. F., Gendelman O. V., Bergman L. A., McFarland D. M., Kerschen G., Lee Y. S., Nonlinear trageted energy transfer in mechanical and structural systems I, Springer, U.S., 2009a.

Vakakis A. F., Gendelman O. V., Bergman L. A., McFarland D. M., Kerschen G., Lee Y. S., Nonlinear trageted energy transfer in mechanical and structural systems II, Springer, U.S., 2009b. 